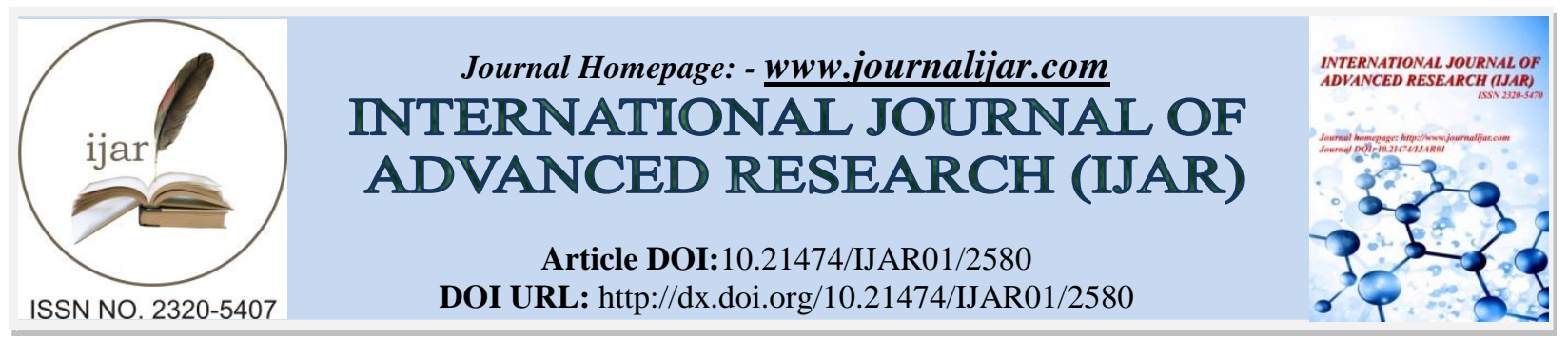

RESEARCH ARTICLE

\title{
SYMPTOMATIC HYPONATREMIA, ETIOLOGY AND OUTCOME IN A TERTIARY CARE HOSPITAL.
}

\author{
Muzaffar Maqbool, Akhter Amin Raina ${ }^{2}$, Bilal A Mir ${ }^{3}$, Asma Rafi ${ }^{3}$ and Parvaiz A Shah ${ }^{4}$. \\ 1. Assistant professor, Department of Medicine, Government Medical College Srinagar, J \& K India. \\ 2. Registrar, Department of Medicine, Government Medical College Srinagar, J \& K India. \\ 3. Post-graduate, Department of Medicine, Government Medical College Srinagar, J \& K India. \\ 4. Professor, Department of Medicine, Government Medical College Srinagar, J \& K India.
}

\section{Manuscript Info}

\section{Manuscript History}

Received: 27 October 2016

Final Accepted: 25 November 2016

Published: December 2016

Key words:-

Hyponatremia, Euvolemic, Diuretics, SIADH.

\section{Abstract}

Background: Hyponatremia is one of the common electrolyte abnormalities in hospitalized patients. The spectrum of different etiologies of hyponatremia have both prognostic and therapeutic implications.

Aims and Objectives: To evaluate the clinical and etiological profile of hyponatremia, and to study the outcome of hyponatremia.

Study Design: A prospective cohort Study.

Materials and Methods: A total of 100 consecutive patients with hyponatremia over a period of one year were enrolled. Detailed clinical history, physical examination, baseline biochemical and metabolic profile followed by serum and urine osmolality and urine sodium was done in all patients.

Results:Out of 100 consecutive patients enrolled in this study, thirtyeight were males and sixty-two were females with male to female ratio of 1:1.6, with mean age of 61.2 years. Commonest neurological complaints were confusion (46\%), lethargy (20\%) and seizures (10\%). Diuretic use was the most common cause of hyponatremia 34\%, followed by SIADH 29\%, gastrointestinal losses $10 \%$ and chronic kidney disease in $8 \%$ of patients. Chronic liver disease and chronic heart failure contributed $6 \%$ each. Other rare causes like hypothyroidism, primary adrenal failure, primary polydipsia and Beer potomania was found in $7 \%$ of patients. $46 \%$ patients were euvolemic, $37 \%$ hypervolemic and $17 \%$ hypovolemic.

Conclusion: Diuretics are the most common cause of hyponatremia followed by SIADH and gastrointestinal losses. Mortality rate in our study was $12 \%$, with maximum mortality(50\%) in SIADH group .

Copy Right, IJAR, 2016,. All rights reserved.

\section{Introduction:-}

Hyponatremia is one of the commonest electrolyte abnormality in hospitalized patients ${ }^{1,2}$, with incidence varying from $1-40 \%$ in different studies. Hyponatremia, defined as serum sodium concentration of $<135 \mathrm{mmol} / \mathrm{l}$ is broadly categorized on the basis of severity into mild hyponatremia (130-134), moderate hyponatremia(120-129) and severe hyponatremia $(<120 \mathrm{mmol} / 1)^{3,4,5}$. Hyponatremia has a wide clinical spectrum varying from anorexia, nausea and 
vomiting to severe symptoms like seizures, coma and death ${ }^{4,6,7}$. Early recognition of hyponatremia decreases morbidity and mortality. The prognostic implications of hyponatremia are grave and far reaching, unless addressed meticulously ${ }^{8}$. This study was conducted to evaluate the etiological profile of admitted patients with symptomatic hyponatremia and their eventual in-hospital outcome.

\section{Aims and objectives:- \\ $>$ To evaluate the clinical and etiological profile of hyponatremia. \\ $>$ To study the in-hospital outcome of hyponatremia.}

\section{Materials and Methods:-}

This study was conducted in the department of medicine in a tertiary care hospital of Kashmir over a period of one year from October 2015 to September 2016. The study was approved and cleared by the ethical committee of the hospital. Thorough history and clinical examination of each individual patient was performed in the ward. Any patient with symptomatic hyponatremia and serum sodium concentration of $<125 \mathrm{meq} / \mathrm{l}$ was included in this study. A detailed history regarding presenting symptoms, course of symptomatology and history regarding co-morbid illness like hypertension and diabetes mellitus was recorded. In patients with co-morbid illness, detailed drug history was noted from their prescription cards with special emphasis on use of diuretics, selective serotonin reuptake inhibitors, and other important medications. Volume status of each individual patient was assessed. Patients were categorized as hypervolemic, hypovolemic or euvolemic depending on the volume status. In hypervolemic patients clinical features ofperipheral edema(like swelling of lower limbs, sacral edema orperi-orbital puffiness), ascites and pleural effusion was noted. Patients having dry tongue, decreased skin turgor and orthostatic hypotension were classified as hypovolemic. Complete hemogram, liver function tests, kidney function tests, blood glucose, serum electrolytes $\left(\mathrm{Na}^{+}, \mathrm{K}^{+}, \mathrm{Ca}^{2+}, \mathrm{PO}_{4}{ }^{2+}, \mathrm{Cl}^{-}\right.$and $\mathrm{HCO}_{3}$ ), routine urine examination, X-ray chest and ultrasonography was performed in all patients. Serum sodium estimation was done in the automated analyzer using ion selective electrode technology. Plasma and urine osmolality was estimated by depression in freezing point method in all patients. Urinary sodium concentration was also checked in each individual patient. MRI brain, CT head, thyroid function and serum cortisol estimation was done as directed by the clinical scenario. Normal serum sodium concentration of $135-145 \mathrm{mmol} / \mathrm{l}$ is the reference range in our laboratory. Patients with serum sodium concentration of $<125 \mathrm{mmol} / 1$ were included in the study. The normal reference range of serum osmolality and urine osmolality in our laboratory is $278-298 \mathrm{mmol} / 1$ and 300-900mmol/1 respectively.Patients with paraproteinemia, hyperlipidemia and those receiving mannitol were excluded from the study.

\section{Statistical analysis:-}

Data was analyzed using EpiInfo 7.0. Relationship between two categorical variables was analyzed using chi-square test.Two sided p-values were reported and a p-value $<0.05$ was considered statistically significant.

\section{Results:-}

A total of 100 consecutive patients with symptomatic hyponatremia $\left(\mathrm{Na}^{+}\right.$concentration $<125$ mmol/l) were included in this study over a period of one year. 62(62\%) were females and 38(38\%) males(Table1).Age range was $16-85$ years with a mean age of 61.2 years. The commonest neurological complaint was confusion $46 \%$, lethargy $20 \%$, stupor $16 \%$, seizures $10 \%$ and coma $8 \%$. Euvolemic hyponatremia was found in 46(46\%) of patients, hypervolemic hyponatremia in $37(37 \%)$ of patients and hypovolemic hyponatremia in $17(17 \%)$ of patients. The most common etiology of hyponatremia in our study was use of diuretics 34(34\%) patients (Table 2). 20 patients with hydrochlorthiazide, 11 patients with chlorthalidone, 2 patients with metalazone and one patient with combination of hydrochlorthiazide and metalazone were hyponatremic. Among the diuretics, chlorthalidone was associated with more severe hyponatremia than other diuretics. The second most common etiology of hyponatremia in our study was SIADH 29(29\%) of patients. 18 patients had cerebrovascular accident (10 patients haemorrhagic stroke, 4 patients sub-arachnoid hemorrhage and 4 patients with ischemic stroke), SSRI use in 6 patients, encephalitis in 3 patients and meningitis in 2 patients. The third most common cause of hyponatremia in our study was gastrointestinal losses in $10(10 \%)$ patients followed by chronic kidney disease $8(8 \%)$, chronic liver disease $6(6 \%)$ and chronic heart failure in $6(6 \%)$ patients. Hypothyroidism was found in $3(3 \%)$ patients, primary adrenal failure in $2(2 \%)$, primary polydipsia in $1(1 \%)$ and Beer potomania in $1(1 \%)$ patient.

Management of the patients was individualized depending on the underlying etiology. Increased salt intake, restriction of total fluid intake of less than a litre per day and hypertonic saline intravenously after calculation of 
proper dose and rate of infusion( $2 \mathrm{meq} / \mathrm{hr})$ not more than $10 \mathrm{meq} / \mathrm{day}$ was instituted. One patient developed central pontine myelinolysis even after slow and proper correction of hyponatremia. 12(12\%) patients with very severe hyponatremia $<110 \mathrm{meq} / \mathrm{l}$ expired in our study.

Table 1:-Demographic and clinical characteristics of study subjects.

\begin{tabular}{|c|c|c|c|}
\hline \multicolumn{2}{|c|}{} & No. of Patients & Frequency(\%) \\
\hline \multirow{3}{*}{ Age (Years) } & $16-25$ & 2 & 2 \\
\cline { 2 - 4 } & $26-35$ & 6 & 6 \\
\cline { 2 - 4 } & $36-45$ & 14 & 14 \\
\cline { 2 - 4 } & $46-55$ & 20 & 20 \\
\cline { 2 - 4 } & $56-65$ & 40 & 40 \\
\cline { 2 - 4 } & $>65$ & 18 & 18 \\
\hline \multirow{4}{*}{ Gender } & Males & 38 & 38 \\
\cline { 2 - 4 } & Females & 62 & 46 \\
\hline \multirow{3}{*}{ Type of Hyponatremia } & Euvolemic & 46 & 37 \\
\cline { 2 - 4 } & Hypervolemic & 37 & 17 \\
\cline { 2 - 4 } & Hypovolemic & 17 & 62 \\
\hline
\end{tabular}

Tabled 2:-Etiology and Outcome of Hyponatremia.

\begin{tabular}{|c|c|c|c|}
\hline & & No. of Patients & Percentage \\
\hline \multicolumn{4}{|c|}{ ETIOLOGY } \\
\hline \multirow[t]{4}{*}{ Diuretics $(n=34)$} & Hydrochlorthiazide & 20 & 20 \\
\hline & Chlorthalidone & 11 & 11 \\
\hline & Metalozone & 2 & 2 \\
\hline & HCTZ+Metalozone & 1 & 1 \\
\hline \multirow[t]{4}{*}{ SIADH $(\mathrm{n}=29)$} & Stroke & 18 & 18 \\
\hline & SSRI intake & 6 & 6 \\
\hline & Encephalitis & 3 & 3 \\
\hline & Meningitis & 2 & 2 \\
\hline \multicolumn{2}{|c|}{ Gastrointestinal Losses } & 10 & 10 \\
\hline \multicolumn{2}{|c|}{ Chronic kidney disease } & 8 & 8 \\
\hline \multicolumn{2}{|c|}{ Chronic liver disease } & 6 & 6 \\
\hline \multicolumn{2}{|c|}{ Congestive heart failure } & 6 & 6 \\
\hline \multicolumn{2}{|c|}{ Hypothyroidism } & 3 & 3 \\
\hline \multicolumn{2}{|c|}{ Primary adrenal failure } & 2 & 2 \\
\hline \multirow{2}{*}{\multicolumn{2}{|c|}{$\begin{array}{c}\text { Primary polydipsia } \\
\text { Beer Potomania }\end{array}$}} & 1 & 1 \\
\hline & & 1 & 1 \\
\hline \multirow[t]{5}{*}{ Mortality } & Stroke & 3 & 3 \\
\hline & Chronic kidney disease & 2 & 2 \\
\hline & Chronic liver disease & 3 & 3 \\
\hline & Congestive heart failure & 3 & 3 \\
\hline & Beer potomania & 1 & 1 \\
\hline
\end{tabular}

Table 3:- Outcome with respect to type of Hyponatremia

\begin{tabular}{|c|c|c|c|}
\hline Type of hyponatremia & Died(n=12) & Survived(n=88) & \multirow{2}{*}{0.049} \\
\hline Euvolemic & 4 & 42 & \\
\hline Hypervolemic & 8 & 29 & \\
\hline Hypovolemic & 0 & 17 & \\
\hline
\end{tabular}

\section{Discussion:-}

Hyponatremia is not a disease itself, but many serious and life threatening conditions can manifest with hyponatremia. It has different etiologies with a variation in frequency of different diseases leading to hyponatremia in different populations groups. 
In the present study of 100 patients, definite female predominance was observed with a male to female ratio of 1:1.6. The mean age of patients was 61.2 years. Similar results were observed by Hochman ${ }^{9}$ and Vurgese ${ }^{10}$ in their study and they had shown that hyponatremia was more common in elderly patients. With advancing age, the development of co-morbidities like hypertension, diabetes, heart failure, chronic kidney disease and use of drugs like diuretics and antidepressants increases, which are known to cause or aggravate hyponatremia.

46\% patients were euvolemic, 37\% patients hypervolemic and 17\% patients were hypovolemic. Most other studies also observed euvolemic hyponatremia as the most common entity except in the study by Hochman ${ }^{9}$ where hypervolemic hyponatremia was more common. Confusion (46\%) and lethargy (20\%) were the most common neurological symptoms as reported by other studies ${ }^{11,12}$. Seizures were present in $10 \%$ of patients. The most common cause of hyponatremia in our study was diuretics $34 \%$ of patients, with hydrochlorothiazide being the most common and chlorthalidone being associated with more severe hyponatremia. Many other studies have also reported that thiazide diuretics are a major cause of hyponatremia especially in elderly ${ }^{6,13,14}$.SIADH was the second most common cause of hyponatremia in our study $29(29 \%)$ of patients, with stroke being the commonest(18 patients) and SSRI use in 6 patients. Hyponatremia was more severe and associated with increased mortality in patients with SIADH. Euvolemic hyponatremia due to SIADH was the most common cause of hyponatremia by $\mathrm{Laczi}^{7}$ in Hungary. The mortality rate in our study was $12 \%$ (12 patients), 4 female patients $(33.3 \%)$ and 8 male patients (66.6\%) (Table 3). Although, hyponatremia was more common in females but females responded to treatment better as compared to males. Higher mortality in elderly hyponatremic patients was observed in past studies ${ }^{15}$. Patients who expired in our study, associated underlying disease like stroke, advanced kidney disease and heart failure were the major contributing factors.

\section{Conclusion:-}

Hyponatremia is one of the common electrolyte abnormality encountered in our day to day clinical practice and with diuretics being used as a preferred choice of antihypertensive agents especially in the elderly, it is becoming common and can occasionally result in increased morbidity and mortality.We recommend that diuretics should be used with caution and in low doses in elderly patients. Close monitoring for serum sodium and potassium should be done in all these patients.

\section{References:-}

1. Upadhyay A, Jaber BL, Madias NE. Incidence and prevalence of hyponatremia. Am J Med. 2006; 119:S30-5.

2. Reddy P, Mooradian AD. Diagnosis and management of hyponatremia in hospitalized patients. Int J CliPract 2009; 63:494-508.

3. Saeed BO, Beaumont D, Handley GH, Weavev J U. Severe hyponatremia: Investigation and management in a district general hospital. J CliPathol. 2002; 55:893-896

4. Heinrich S, Wagner A, Gross P. Hyponatremia. Med Klinn Intensive Med Notfmed. 2013; 108(1):53-8.

5. Shannon G. Severe hyponatremia- recognition and management. Australian Prescriber 2011;34(2).

6. Clayton J. A. Severe hyponatremia in medical inpatients: etiology, assessment and outcome. Q J Med. 2006; 99: 505-511.

7. Laczi F. Etiology. Diagnostics and therapy of hyponatremias. Orv Hetil. 2008; 149(29): 1347-54.

8. Waiker SS, Mount DB, Curhan GC. Mortality after hospitalization with mild, moderate, and severe hyponatremia. Am J Med 2009; 122:857-65.

9. Hochman I, Cabili S, Peer G. Hyponatremia in internal medicine ward patients: causes, treatment and prognosis. Isr J Med Sci. 1989;25(2): 73-6.

10. Vurgese TA, Radha Krishan SB, Mapkar OA. Frequency and etiology of hyponatremia in adult hospitalized patients in medical wards of a general hospital in Kuwait. Kuwait Medical Journal. 2006;38(3):211-3.

11. Rao MY, Sudhir U, anil Kumar T, Saravanan S, Mahesh E, Punith K. Hospital based descriptive study of symptomatic hyponatremia in elderly patients. J Asssoc Physicians India. 2010:667-9.

12. Nandkumar, Gane B, Hiremath PB. Clinico-aetiological profile of hyponatremia in adults. Int J Bio Med Res. 2013;4(1):2802-6.

13. Adrogue H, MadiasN.Hyponatremia. N Engl J Med 2000; 342: 1581-1589.

14. Reynolds RM, Padfield PL, Seckl JR. Disorders of sodium balance. BMJ 2006;332:702-5.

15. StemsRH. Severe symptomatic hyponatremia: treatment and outcome. A study of 64 cases. Ann Intern Med 1987;107:656-664. 